\title{
The Role of DNA Methylation in Epigenetic Control of Antibiotic Resistance Genes
}

ISSN: 2637-8078

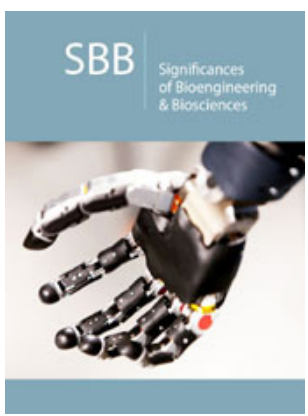

*Corresponding author: Fahad Al Mathkhury HJ, Department of Biology, College of Science, Iraq

Submission: 海June 15, 2019

Published: 㘹July 22, 2019

Volume 3 - Issue 4

How to cite this article: Fahad Al Mathkhury HJ. The Role of DNA Methylation in Epigenetic Control of Antibiotic Resistance Genes. Significances Bioeng Biosci.3(4). SBB.000567.2019.

DOI: 10.31031/SBB.2019.03.000567

Copyright@ Fahad Al Mathkhury HJ, This article is distributed under the terms of the Creative Commons Attribution 4.0 International License, which permits unrestricted use and redistribution provided that the original author and source are credited.

\author{
Fahad Al Mathkhury HJ*
}

Department of Biology, Iraq

\section{Exaptation}

The evolution of antibiotic resistance in bacteria is an issue of great importance. Antibioticresistant strains are continuously emerging, and, currently, bacterial and viral infections are responsible for approximately $5-10 \%$ of deaths in industrialized world; more than $30 \%$ of deaths in Southeast Asia, and 60\% in Africa [1].

Clearly, studying the emergence and dissemination of antibiotic resistance is important with respect to human health and is one of the potential applications of following evolution in real time. It has been reported that the resistance genes picked up by pathogens through horizontal gene transfer (HGT) probably originated in antibiotic-producing bacteria, in which they played a self-protection role [2]. The function of antibiotic resistance genes is, therefore, the same in both the original and new host. Whether this theory holds true for genes conferring resistance to synthetic antibiotics such as quinolones not well understood. The quinolones are a family of broadly used synthetic antimicrobial agents that target bacterial topoisomerases (DNA gyrase and topoisomerase IV) and thereby inhibit bacterial DNA replication and transcription [3]. Due to their synthetic origin, it was hypothesized that no naturally occurring quinolone resistance genes exist; rather, antibiotic resistance should arise through mutations in the genes encoding quinolone targets only.

Nevertheless, quinolones are one of the most common substrates of multidrug (MDR) efflux pumps, which are chromosomally encoded in all bacterial species, indicating that microorganisms have genes involved in quinolone resistance despite the synthetic origin of these drugs [4]. Therefore, quinolone resistance is a good model to help us understand the role of epigenetics in drug resistance. Quinolone resistance (qnr) genes must have originated in bacteria that do not produce quinolones (since there are no quinolone producers in nature), therefore the original function of these genes could not have been quinolone resistance. This observation raises a significant evolutionary question: what evolutionary process, permitted the conversion of a gene not involved in quinolone resistance into one that does confer quinolone resistance? Specifically, has the exposure of bacteria to quinolones affected the evolution of such otherwise any gene with primary function in the cell other than conferring antibiotic resistance (e.g. housekeeping genes)? Furthermore, given that there are some qnr genes that can potentially be transferred to a heterologous host [5] why do only a few of them exist in the plasmids found in human pathogens? Pre-resistance genes that act on chemical moieties analogous to antibiotics may be the answer. These pre-resistance genes would not confer resistance originally but might effortlessly evolve under antibiotic selective pressure to result in a bona fide antibiotic resistance determinant [6]. Alternatively, these genes may be able to confer resistance without any modification at all [7]. This latter category of evolution, in which the function of a given gene is altered as a result of changes in the environment, rather than changes in the sequence of the gene encoding it, has been termed exaptation [8]. In the case of antibiotic resistance, exaptation relates to the metabolic and regulatory de-contextualization pre-resistance genes undergo when jumping from the original host 
chromosome to that of a novel host with different metabolism and regulatory networks [9]. DNA methylation is one of several possible factors involved in this 'shift of function' to antibiotic resistance without change in gene sequence of the pathogen in question.

\section{DNA methylation: what is it?}

DNA methylation can be defined as the covalent addition of a methyl group $\left(-\mathrm{CH}_{3}\right)$ to cytosine (C) dinucleotides (methylated cytosine residues are sometimes referred to as the fifth nucleotide). DNA methylation plays an essential role in gene expression regulation and, generally but not always, it is associated with loss of gene expression [10]. One theory on the evolution of DNA methylation is that it evolved as a host defense mechanism to silence foreign DNA, such as viral sequences, replicated transposable elements, and other repetitive sequences [11]. Here we consider its role in gene expression.

\section{DNA methylation and gene expression?}

DNA methylation can prevent gene expression directly or indirectly through restriction of access of transcription factors to the gene promoter, which must be accessible to the several enhancers of transcription for normal function. Therefore, DNA methylation appears to be one of several mediators of what we observe as epigenetic inheritance. In the context of evolution of antibiotic resistance, changes in DNA methylation status could modulate the expression of antibiotic resistance genes directly, by reducing the methylation of resistance genes and thereby increasing their expression. Alternatively, increasing the methylation status of a gene that represses the function of a resistance gene to reduce its expression would de-repress the resistance gene and thereby indirectly promote its expression. A particular example of DNA methylation's role in antibiotic resistance genes such as flu is provided by Henderson et al. [12] In this case, the deoxyadenosine methyltransferase (DAM) of $E$. coli methylates an adenine sequence which occurs approximately 18,000 times in the E. coli genome. Here, the new strand of DNA product is also methylated by DAM, thus preserving the change in function without a change in coding in the next generation. Epigenetic memory mechanisms are, thus, capable of preserving the gene expression state in bacteria for many generations; transcriptional memory is apparently mediated by the same epigenetic mechanisms as were the original phenotypic changes. Therefore, expanding our understanding of these complex epigenetic mechanisms which drive antibiotic resistance is both of practical relevance and of critical importance as we enter what has been called the "post-antibiotic era".

\section{Summary}

Microbial infections are responsible for a significant proportion of deaths worldwide, and microbes are continuously developing and disseminating drug resistance. Under antibiotic pressure, pre-resistance genes can easily evolve to result in new antibiotic resistance determinants Furthermore, some genes that confer functional roles in resistance may also encode resistance without any modification. Alternatively, the expression of resistance genes can be regulated via epigenetic mechanisms such as DNA methylation, which emphasized the role of evolutionary variation in developing antibiotic resistance. Given that phenotypic resistance does not necessarily reflect a presence of an encoding gene.

\section{References}

1. Adam M, Murali B, Glenn NO, Potter S (2008) Epigenetic inheritancebased evolution of antibiotic resistance in bacteria. BMC Evolutionary Biology 8: 52-63.

2. Laskaris P, Tolba S, Bado CL, Wellington L (2010) Coevolution of antibiotic production and counter-resistance in soil bacteria. Environmental Microbiology 12(3): 783-796.

3. Hernandez A, Sanchez M, Martinez J (1999) Quinolone resistance: Much more than predicted. Front Microbiol 2: 22.

4. Alonso A, Rojo F, Martinez J (1999) Environmental and clinical isolates of Pseudomonas aeruginosa show pathogenic and biodegradative properties irrespective of their origin. Environ Microbiol 1(5): 421-430.

5. Sanchez M, Hernandez A, Martinez RJ, Martinez ML, Martinez J (2008) Predictive analysis of transmissible quinolone resistance indicates Stenotrophomonas maltophilia as a potential source of a novel family of Qnr determinants. BMC Microbiol 8.

6. Aminov R (2009) The role of antibiotics and antibiotic resistance in nature. Environ Microbiol 11(12): 2970-2988.

7. Baquero F, Ortega AC, Martinez JL (2009) Ecology and evolution of antibiotic resistance. Environ Microbiol Reports 1(6): 469-476.

8. Gould S, Vrba S (1982) Exaptation: A missing term in the science of form. Paleobiology 8(1): 4-15.

9. Sanchez MB, Martinez JL (2012) Differential epigenetic compatibility of qnr antibiotic resistance determinants with the chromosome of Escherichia coli. PLoS ONE 7: 10.

10. Bird AP (1986) CpG-rich islands and the function of DNA methylation. Nature 321(6067): 209-213.

11. Hedges DJ, Batzer MA (2005) From the margins of the genome: Mobile elements shape primate evolution. Bioessays 27: 785-794.

12. Henderson IR, Owen P (1999) The major phase-variable outer membrane protein of Escherichia coli structurally resembles the immunoglobulin A1 protease class of exported protein and is regulated by a novel mechanism involving dam and oxyR. J Bacteriol 181(7): 21322141. 